\title{
Differential detection of cytoplasmic Wilms tumor 1 expression by immunohistochemistry, western blotting and mRNA quantification
}

\author{
TAKEHIRO MAKI ${ }^{1}$, HIROAKI IKEDA $^{2}$, AKI KURODA $^{3}$, NORIAKI KYOGOKU ${ }^{1}$, YOSHIYUKI YAMAMURA ${ }^{1}$, \\ YUKIKO TABATA $^{3}$, TAKEHIRO ABIKO ${ }^{1}$, TAKAHIRO TSUCHIKAWA ${ }^{1}$, YASUHIRO HIDA ${ }^{3}$, \\ TOSHIAKI SHICHINOHE ${ }^{1}$, EIICHI TANAKA ${ }^{1}$, KICHIZO KAGA ${ }^{3}$, KANAKO HATANAKA ${ }^{4}$, \\ YOSHIHIRO MATSUNO ${ }^{4}$, NAOKO IMAI ${ }^{5}$ and SATOSHI HIRANO ${ }^{1}$ \\ ${ }^{1}$ Department of Gastroenterological Surgery II, Hokkaido University Graduate School of Medicine, Sapporo, Hokkaido, \\ 060-8638; ${ }^{2}$ Department of Oncology, Nagasaki University Graduate School of Biomedical Sciences, Nagasaki 852-8523; \\ ${ }^{3}$ Department of Cardiovascular and Thoracic Surgery, Hokkaido University Graduate School of Medicine, Sapporo, \\ Hokkaido 060-8638; ${ }^{4}$ Department of Surgical Pathology, Hokkaido University Hospital, Sapporo, Hokkaido 060-8648; \\ ${ }^{5}$ Department of Immuno-gene Therapy, Mie University Graduate School of Medicine, Mie 514-8507, Japan
}

Received September 14, 2016; Accepted November 14, 2016

DOI: $10.3892 /$ ijo.2016.3786

\begin{abstract}
Wilms tumor 1 (WT1) is considered to be a promising target of cancer treatment because it has been reported to be frequently expressed at high levels in various malignancies. Although WT1-targeted cancer treatment has been initiated, conclusive detection methods for WT1 are not established. The present study aimed to consolidate immunohistochemistry for WT1 with statistical basis. Transfected cells with forced WT1 expression yielded specific western blot bands and nuclear immunostaining; cytoplasmic immunostaining was not specifically recognized. Immunohistochemistry, western blotting, and quantitative reverse transcriptase-polymerase chain reaction were performed in 35 human cell lines using multiple WT1 antibodies and their results were quantified. Relationships among the quantified results were statistically analyzed; the nuclear immunostaining positively correlated with western blot bands and mRNA expression levels, whereas cytoplasmic immunostaining did not. These results indicate
\end{abstract}

Correspondence to: Dr Takehiro Maki, Department of Gastroenterological Surgery II, Hokkaido University Graduate School of Medicine, North 15, West 7, Kita-ku, Sapporo, Hokkaido 060-8638, Japan

E-mail: qwerty75568@yahoo.co.jp

Abbreviations: WT1, Wilms tumor 1; cDNA, complementary DNA; GFP, green fluorescent protein; PCR, polymerase chain reaction; qRT-PCR, quantitative reverse transcriptase-polymerase chain reaction; EN, entire nucleus; NB, nuclear bodies; $\mathrm{CP}$, cytoplasm; IC, intercalator-base method; FP, fluorescent probe-based method; SCC, squamous cell carcinoma

Key words: immunoblotting, immunohistochemistry, neoplasms, reverse transcriptase polymerase chain reaction, Wilms Tumor 1 protein that nuclear immunostaining reflects WT1 expression but cytoplasmic immunostaining does not. The nuclear immunostaining was barely (3/541) observed in primary cancer of esophagus, bile duct, pancreas and lung. Although the present study has some limitations, the results indicate that the cytoplasmic immunostaining does not correlate with actual WT1 expression and prompts researchers to carefully evaluate target molecule expression in treatment of cancer.

\section{Introduction}

The Wilms tumor 1 (WT1) gene was initially identified in 1990 as a tumor-suppressor gene of Wilms tumor $(1,2)$. WT1, which contains four C-terminal zinc-finger motifs and an $\mathrm{N}$-terminal DNA-binding domain, functions as a transcription factor in regulation of differentiation and development of mesodermally derived tissues such as kidney, mesothelium and gonad (3-5). WT1 has been reported to be strongly expressed in benign mesodermal tissues, as well as in malignancies of mesodermal origin such as leukemia (44-93\%) $(6,7)$, mesothelioma (72-100\%) (8-14) and ovarian serous carcinomas $(45-100 \%)(11,13,15,16)$. Therefore, WT1 mRNA is used as a clinical standard marker to diagnose minimal residual disease of leukemia (6) and immunohistochemical study for WT1 is helpful to distinguish mesodermally derived solid cancers such as mesothelioma and ovarian cancer from other solid cancers $(8,10-13,15,16)$.

On the other hand, WT1 attracts many researchers as an ideal target for cancer treatment. WT1 was prioritized as a promising target of immunotherapy against various malignancies (17) because dozens of studies have confirmed WT1 overexpression not only in mesodermally derived malignancies but also in a variety of non-mesodermal origin solid cancers such as esophageal $(45-95 \%)(18,19)$, gastric $(42 \%)(19)$, colon $(69-89 \%)(19,20)$, hepatocellular $(95 \%)(21)$, bile duct $(68 \%)$ (19), pancreatic $(65-75 \%)(19,22)$, thyroid $(95 \%)(23)$, prostate (25\%) (9), lung (30-83\%) $(19,24)$, breast $(26-87 \%)(19,25-27)$, 
and brain cancers $(88-96 \%)(19,28)$. These solid tumors do not carry mutations in the WT1 gene $(20,23,24,28)$. Based on these results, clinical trials of WT1-specific immunotherapy for patients with various tumors have been conducted (29-36).

In solid cancers, WT1 expression is generally confirmed by immunohistochemistry but methods of the immunohistochemistry and interpretations of the results are diverse among studies although they are essential to appropriate clinical trials of WT1-specific treatment. In the present study, we aimed to establish reasonable interpretations of results of immunohistochemistry for WT1 and to re-evaluate WT1 expression in primary esophageal, bile duct, pancreatic and lung cancers.

\section{Materials and methods}

Cell lines. Thirty-five human cell lines were used, derived from the following tissue or tumor sources: 293FT, embryonal kidney; K562, chronic myelogenous leukemia; HMMME, ACC-MESO-1 and ACC-MESO-4, mesothelioma; JHOS-2 and JHOS-3, ovarian serous adenocarcinoma; HeLa, uterine cervical cancer; TE-2, TE-4, TE-5, TE-8, TE-9, TE-14, SGF7 and HEC46, esophageal cancer; TFK-1, HuCCT1, TKKK, RBE and HuH-28, bile duct cancer; PANC-1, PCI-6, KP-1N, SUIT-2, AsPC-1 and BxPC-3, pancreatic cancer; A549, RERF-LC-MS, RERF-LC-OK, ABC-1 and VMRC-LCD, lung adenocarcinoma; H226, LK-2 and PC10, lung squamous cell carcinoma.

293FT was purchased from Invitrogen (Carlsbad, CA, USA). K562, HMMME, ACC-MESO-1 (37), ACC-MESO-4 (37), JHOS-2, JHOS-3, HeLa, TFK-1, HcCCT1, TKKK, RBE, HuH-28 and PANC-1 were purchased from the RIKEN BioResource Center Cell Bank (Tsukuba, Japan). The TE series was provided by Dr T. Nishihira (Tohoku University, Sendai, Japan) (38). SGF7 was provided by Dr T. Saito (Toyama Medical and Pharmaceutical University, Toyama, Japan) (39). HEC46 was provided by Dr T. Toge (Hiroshima University, Hiroshima, Japan) (40). PCI-6 was provided by the First Department of Pathology, Hokkaido University (Sapporo, Japan) (41). KP-1N, SUIT-2, A549, RERF-LC-MS, RERF-LC-OK, ABC-1, VMRC-LCD, H226, LK-2 and PC10 were purchased from the Japanese Cancer Research Resource Bank (Tokyo, Japan). AsPC-1 and BxPC-3 were provided by the American Type Culture Collection (ATCC; Manassas, VA, USA).

293FT was cultured in Dulbecco's modified Eagle's medium (Sigma-Aldrich, St. Louis, MO, USA). The other cell lines were cultured in RPMI-1640 (Sigma-Aldrich). Each medium was supplemented with $10 \%$ fetal bovine serum (FBS) and $1 \%$ penicillin/streptomycin. All cell lines were cultured at $37^{\circ} \mathrm{C}$ in a humidified atmosphere containing $5 \% \mathrm{CO}_{2}$. Trypsin $(0.25 \%)$ was used for subculture.

Tissue samples. Cancer tissues and corresponding normal epithelial tissues were obtained from 552 patients with esophageal (101 patients), bile duct (96 patients), pancreatic (99 patients), and lung cancer (256 patients) who underwent resection in the Department of Gastroenterological Surgery II, Hokkaido University, Japan between 1994 and 2005. These tissue samples were fixed in $10 \%$ formalin and embedded in paraffin blocks. Three or four spots of each tissue, of uniform

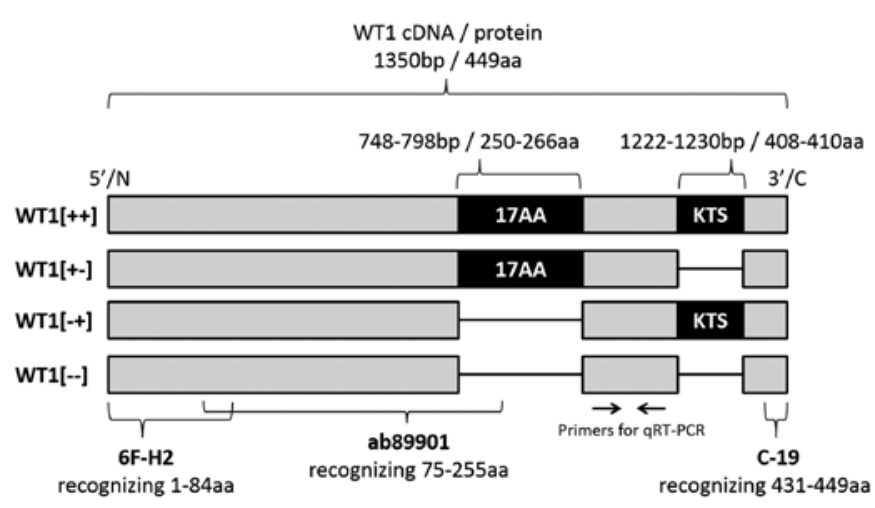

Figure 1. WT1 splice variants and WT1-reactive antibodies used in the present study. Four splice variants of WT1 were designated as $[++],[+-],[-+]$ and [--] according to the presence or absence of 17AA region corresponding to all sequence of the exon 5 or KTS region corresponding to nine base pairs of the C-terminus of exon 9, as indicated. The recognition sites of WT1reactive antibodies 6F-H2 (mouse monoclonal), ab89901 (rabbit monoclonal) and C-19 (rabbit polyclonal) are indicated. Bp, base pairs; aa, amino acids.

0.6-mm diameter, were punched out and consolidated into 25 paraffin blocks using a Manual Tissue Arrayer (Beecher Instruments, Inc., Sun Prairie, WI, USA); the resultant 25 blocks were used for immunohistochemistry. As possible positive controls for WT1 expression in tissue samples, kidney, pleura, testis, pleural mesothelioma and ovarian serous adenocarcinoma were also examined under the same conditions.

Gene cloning and transfection. Negative and positive controls for WT1 protein expression were generated as follows. First, internal ribosome entry site and complementary DNA (cDNA) encoding green fluorescent protein (GFP) were cloned into the multiple cloning site of plasmid vector pcDNA3.1(+) (Invitrogen); the resultant plasmid is termed empty vector. WT1 cDNA was amplified by polymerase chain reaction (PCR), using human kidney QUICK-Clone cDNA (Takara, Otsu, Japan) as a template and cloned into empty vector. The resultant plasmid vector is termed vector WT1. The base sequence of vector WT1 was confirmed by the singlenucleotide primer extension method. Empty vector and vector WT1 were transfected into 293FT and HeLa cells using Lipofectamine 2000 (Invitrogen). Successful transfection was verified by observing GFP expression. Cells transfected with empty vector and vector WT1 were, respectively, used as negative and positive controls for WT1 protein expression. The WT1 mRNA contains two splice sites that can be skipped during RNA splicing; consequently, the WT1 gene encodes four variant isoforms of WT1 protein (Fig. 1). We constructed the corresponding four types of positive controls for WT1 protein expression.

Transfected cells producing fusion protein GFP-WT1 were generated as follows. GFP cDNA was cloned into the multiple cloning site of pcDNA3.1(+); the resultant plasmid is termed vector GFP. WT1 cDNA was cloned into vector GFP such that the WT1 cDNA sequence was fused to the C-terminus of the GFP cDNA, which lacked a stop codon; the resultant plasmid is termed vector GFP-WT1. The base sequence of vector GFP-WT1 was confirmed by the single-nucleotide primer extension method. Vector GFP and vector GFP-WT1 were 


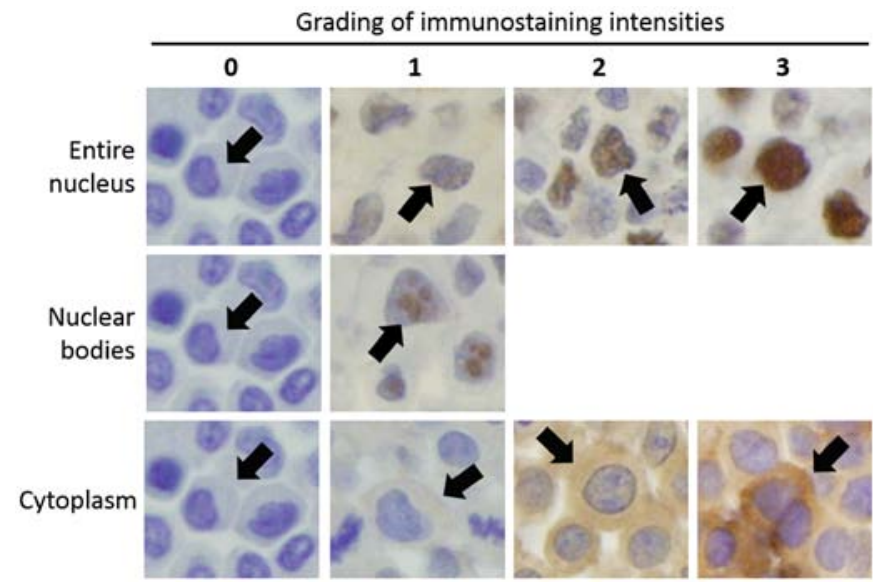

Figure 2. Grading of immunostaining intensities. Grading of immunostaining intensities was defined independently in the entire nucleus, nuclear bodies and cytoplasm. Arrows indicate the cells with representative immunostaining intensities corresponding to each grade.

transfected into 293FT and HeLa cells using Lipofectamine LTX (Invitrogen). Cells transfected with vector GFP and vector GFP-WT1 were predicted to produce GFP and fusion protein GFP-WT1, respectively. As noted above, the WT1 mRNA has four splicing variants (Fig. 1); therefore, we constructed the corresponding four types of vector GFP-WT1 plasmids.

Immunohistochemistry. Cultured cells which were detached using $0.25 \%$ trypsin and sedimented by centrifugation or excised tissue samples were fixed in $10 \%$ formalin and embedded in paraffin blocks. Thin sections ( $2 \mu \mathrm{m}$ thick) were de-waxed and rehydrated, and antigens were retrieved in pressure vessels under the following conditions: citrate buffer ( $\mathrm{pH} 7.0), 2 \mathrm{~atm}, 100^{\circ} \mathrm{C}, 2 \mathrm{~min}$. For additional antigen retrieval, sections for WT1 antibody $6 \mathrm{~F}-\mathrm{H} 2$ were incubated for $5 \mathrm{~min}$ at room temperature with $10 \%$ proteinase $\mathrm{K}$ ready-to-use enzyme (Dako, Kyoto, Japan) before treatment in the pressure vessels. The sections were immersed for $15 \mathrm{~min}$ at room temperature in $0.3 \% \mathrm{H}_{2} \mathrm{O}_{2}$ diluted with methanol, in order to block endogenous peroxidase activity, and then incubated in $10 \%$ normal goat serum (Nichirei Corp., Tokyo, Japan) at room temperature for $30 \mathrm{~min}$ to reduce non-specific binding. WT1 antibodies 6F-H2 (mouse monoclonal; Dako), ab89901 (rabbit monoclonal; Abcam, Cambridge, UK) and C-19 (rabbit polyclonal; Santa Cruz Biotechnology, Dallas, TX, USA), used as primary antibodies (Fig. 1), were diluted 1:100, 1:200 and 1:100, respectively, with antibody diluent (Dako). The sections were incubated with the diluted primary antibodies at $4{ }^{\circ} \mathrm{C}$ overnight. Mouse IgG1 (Dako) and rabbit polyclonal IgG (Abcam) were used for negative controls against samples with primary antibodies. Next, sections were incubated for $30 \mathrm{~min}$ at room temperature with biotinylated goat antibody to mouse and rabbit immunoglobulin (Histofine Simple Stain MAX PO MULTI; Nichirei Corp.). Finally, sections were stained with 3-3'-diaminobenzidine tetrahydrochloride (Histofine Simple Stain DAB Solution; Nichirei Corp.) and then lightly counterstained by hematoxylin.

In cell lines, immunostaining scores for the entire nucleus, the nuclear bodies, and the cytoplasm were calculated independently. Immunostaining intensities of entire nucleus and
Table I. Two patterns of experimental conditions used in western blotting.

\begin{tabular}{|c|c|c|c|c|c|c|}
\hline \multirow{3}{*}{$\begin{array}{l}\text { Primary } \\
\text { antibody }\end{array}$} & \multirow{2}{*}{\multicolumn{2}{|c|}{$\begin{array}{l}\text { Amount } \\
\text { of protein } \\
\text { per lane } \\
(\mu \mathrm{g})\end{array}$}} & \multicolumn{4}{|c|}{ Dilution factor } \\
\hline & & & \multicolumn{2}{|c|}{$\begin{array}{l}\text { Primary } \\
\text { antibody }\end{array}$} & \multicolumn{2}{|c|}{$\begin{array}{l}\text { Secondary } \\
\text { antibody }\end{array}$} \\
\hline & A & B & A & B & A & B \\
\hline \multicolumn{7}{|l|}{ WT1 antibodies } \\
\hline $6 \mathrm{~F}-\mathrm{H} 2$ & 10 & 20 & 40 & 15 & 4000 & 1500 \\
\hline ab89901 & 10 & 20 & 4000 & 500 & 10000 & 7000 \\
\hline C-19 & 10 & 20 & 2000 & 150 & 10000 & 10000 \\
\hline GFP antibody & 10 & 40 & 300 & 50 & 10000 & 300 \\
\hline Actin antibody & \multicolumn{2}{|c|}{5} & \multicolumn{2}{|c|}{800} & \multicolumn{2}{|c|}{10000} \\
\hline
\end{tabular}

Condition A and Condition B were predicted to provide weaker and stronger bands, respectively. Western blotting using the actin antibody was performed under a uniform condition throughout the study. A, Condition A; B, Condition B.

cytoplasm in individual cells were classified into four grades and intensity of nuclear bodies was classified into two grades as shown in Fig. 2. Immunostaining scores for samples were calculated as the sum of the immunostaining intensities of individual cells, divided by the total number of the cells; thus, the score represents the average immunostaining intensity of all cells in the sample. Immunostaining scores were independently calculated by two surgeons under the guidance of a pathologist and the average value was regarded as the final immunostaining score.

Western blotting. Total protein was extracted from cultured cells using triple-detergent lysis buffer. Protein concentration was measured by the Bradford method using a commercial protein assay kit (Bio-Rad Laboratories, Hercules, CA, USA). Protein was boiled for 2 min for antigen retrieval, subjected to SDS-PAGE, and blotted onto a Hybond-ECL nitrocellulose membrane (GE Healthcare Life Sciences, Piscataway, NJ, USA). The membrane was blocked at $4^{\circ} \mathrm{C}$ overnight with $5 \%$ skim milk, incubated with diluted primary antibodies at room temperature for $1 \mathrm{~h}$, and then incubated with diluted secondary antibodies at room temperature for $1 \mathrm{~h}$. WT1 antibodies 6F-H2, ab89901 and C-19 (Fig. 1), mouse monoclonal GFP antibody (Clontech Laboratories, Inc., Otsu, Japan) and actin C4 antibody (Millipore, Billerica, MA, USA) were used as primary antibodies. Peroxidase-conjugated AffiniPure Goat AntiMouse IgG $\mathrm{H}+\mathrm{L}$ antibodies (Jackson ImmunoResearch, West Grove, PA, USA) or peroxidase-conjugated AffiniPure Goat Anti-Rabbit IgG H+L antibodies (Jackson ImmunoResearch) were used as secondary antibodies. Protein bands were visualized using the ECL-Plus Western blotting detection system (GE Healthcare Life Sciences) and Detector Lumino Imaging Analyzer model FAS-1000 (Toyobo, Co., Ltd., Osaka, Japan). Intensities of protein bands were quantitated using ImageJ (http://rsbweb.nih.gov/ij/), corrected for the intensities of the corresponding actin bands, and normalized to the intensity 


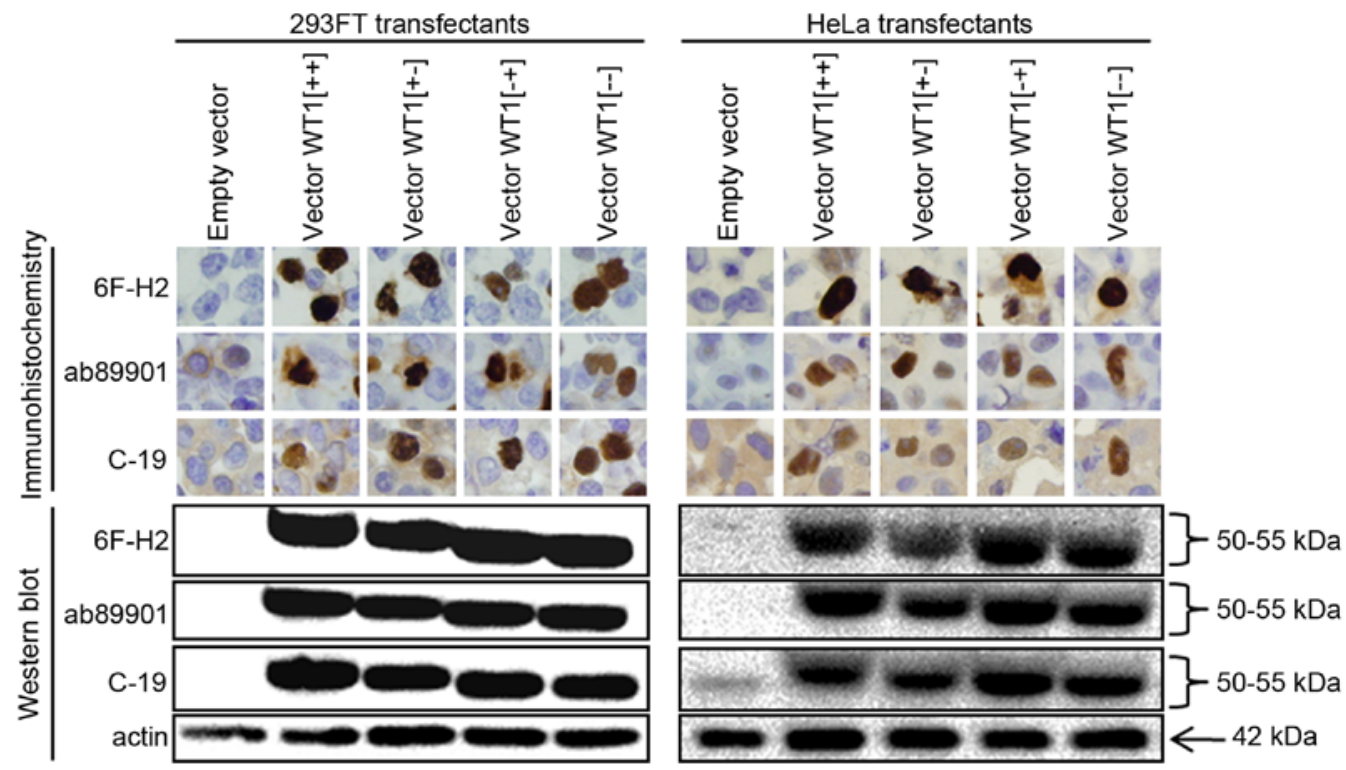

Figure 3. Immunohistochemical and western blot analyses of WT1 protein in transfected cells. Western blotting and immunohistochemistry were performed on 293FT and HeLa cells transfected with four splice variants of WT1, using two monoclonal antibodies (6H-H2 and ab89901) and one polyclonal antibody (C-19). Western blotting for transfected 293FT and HeLa cells was done under Condition A and B, respectively (as described in Table I).

of the protein band obtained from vector WT1[--] transfectants (defined as 1). Two patterns of experimental conditions, which differed in the amount of protein applied to each gel lane and the dilution factors of the antibodies (Table I), were used. However, the intensities of protein bands were compared under the same conditions.

Quantitative reverse transcriptase-polymerase chain reaction ( $q R T-P C R)$. Total RNA was extracted from cultured cells using the TRI reagent (Sigma-Aldrich). Possible DNA contamination was eliminated using RQ1 RNase-Free DNase (Promega, Tokyo, Japan). After the DNase treatment, the absence of DNA contamination was confirmed by PCR using the primers for $\beta$-actin (described below). cDNA was synthesized from mRNA by reverse transcription reaction using the SuperScript VILO cDNA Synthesis kit (Invitrogen). cDNA diluted 20-fold with double-distilled water was used as a template for quantitative PCR. Quantitative PCR was performed by two methods: the intercalator-based method using Power SYBR-Green PCR Master Mix (Applied Biosystems, Tokyo, Japan) and the fluorescent probe-based method using TaqMan Universal Master Mix II (Applied Biosystems). Reaction conditions consisted of 40 cycles of $94^{\circ} \mathrm{C}$ for $30 \mathrm{sec}, 60.4^{\circ} \mathrm{C}$ for $30 \mathrm{sec}$ and $72^{\circ} \mathrm{C}$ for $30 \mathrm{sec}$. All reactions were performed in triplicate. The results of quantitative PCR were analyzed on an ABI PRISM 7000 Sequence detection system (Applied Biosystems). Expression levels of WT1 mRNA were normalized to the corresponding $\beta$-actin mRNA and relativized considering those of WT1 mRNA of K562 as 1.

The base sequences of primers and internal probes were as follows: WT1 sense primer, 5'-TGCGGAGCCCAATACA GAATACAC-3' and WT1 reverse primer, 5'-TCAGATGCCG ACCGTACAAGAG-3'; WT1 internal probe, 5'-FAM-AGA GGCATTCAGGATGTGCGACG-TAMRA-3'; $\beta$-actin sense primer, 5'-CAACCGCGAGAAGATGACCC-3' and $\beta$-actin reverse primer, 5'-ACCGGAGTCCATCACGATGC-3'; $\beta$-actin internal probe, 5'-FAM-CCAGGCTGTGCTATCCCTGTA CGC-TAMRA-3'. The primers for WT1 were designed to bind to WT1 cDNA between exons 6 and 7, so that the four spliced variants of WT1 cDNA were all recognized (Fig. 1). The primers for $\beta$-actin were designed to bind to $\beta$-actin cDNA between exons 3 and 4 .

Statistical analysis. Correlation of paired quantitative variables was evaluated by the Spearman rank method using StatView version 5.0 software (SAS Institute, Inc., Cary, NC, USA). Differences were considered significant when $r>0.4$ and $\mathrm{P}<0.05$.

\section{Results}

WT1 expression in transfected cells. We transfected 293FT and HeLa with vectors encoding the four splice variants of WT1 (Fig. 1). WT1-transfected cells were expected to produce excessive exogenous WT1 protein. As shown in Fig. 3, western blotting with two WT1-specific monoclonal antibodies $(6 \mathrm{~F}-\mathrm{H} 2$ and ab89901) and one WT1-reactive polyclonal antibody (C-19) yielded clear 50-55-kDa bands in cells expressing any of the four WT1 variants, even though the antibodies recognize different sites (Fig. 1). Immunohistochemistry with these three antibodies resulted in strong staining in the nucleus of all WT1 transfectants, corresponding to the appearance of the $50-55-\mathrm{kDa}$ bands in western blotting. In addition to the nuclear staining, these antibodies yielded modest cytoplasmic staining in various cells including empty-vector transfectants. Especially, C-19 yielded significant cytoplasmic immunostaining in all tested cells, regardless of their transfection status. In conclusion, enforced WT1 expression was detected in the nucleus of the two different cell lines by the three different antibodies. Polyclonal C-19 antibody may exhibit non-specific as well as specific reactivity. We used these transfectants as positive and negative controls for further analyses. 

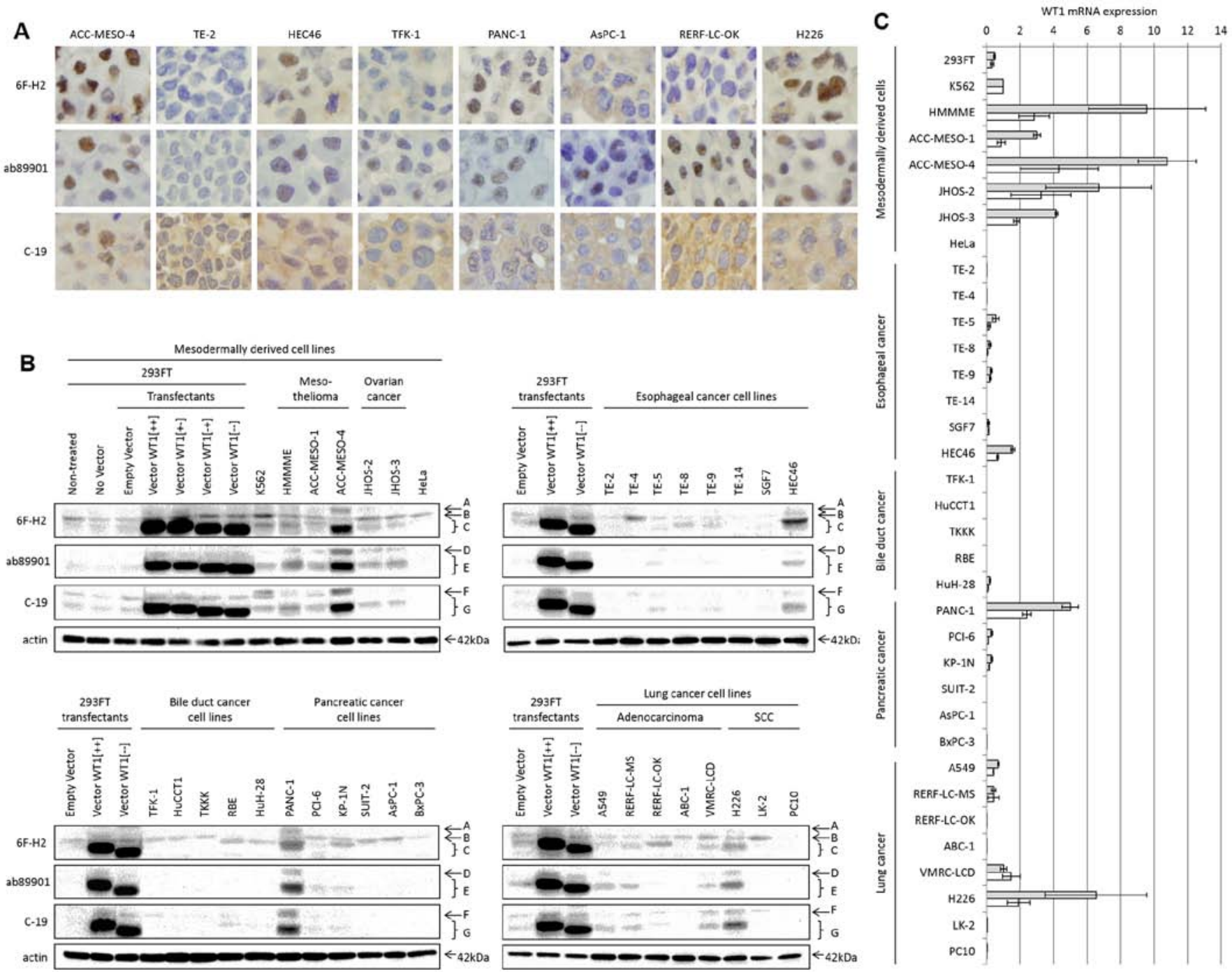

Figure 4. Immunohistochemistry, western blotting and qRT-PCR for WT1 in 35 human cell lines. (A) Representative immunohistochemical images of human cell lines. (B) Results of western blotting for 35 cell lines. Western blotting in this panel was done under Condition B (as described in Table I). 'Non-treated' indicates protein extracted from host cells not subjected to any treatment. 'No vector' indicates protein extracted from host cells subjected to mock transfection (i.e., transfection reagent but no plasmids). 'Vector WT1' indicates the $1 \mu \mathrm{g}$ of protein from vector WT1 transfectants mixed with $19 \mu \mathrm{g}$ of protein from empty vector transfectants. Western blot bands of 60,57 and 50-55 kDa detected by $6 \mathrm{~F}-\mathrm{H} 2$ were named A, B and C, respectively; bands of 60 and $50-55 \mathrm{kDa}$ detected by ab89901, D and E; bands of 60 and 50-55 kDa detected by C-19, F and G. SCC, squamous cell carcinoma. (C) Results of qRT-PCR for 35 cell lines. WT1 mRNA expression was normalized to the corresponding $\beta$-actin mRNA level and further normalized to the level of WT1 mRNA in K562 (defined as 1). Gray and white bars represent the average values of the relative quantities of WT1 mRNA detected by the intercalator-based method and the fluorescent probe-based method, respectively. Error bars represent standard deviation.

Comparison of the results of WT1-immunohistochemistry with those of other detection methods using cell lines. By itself, immunohistochemistry does not distinguish specific from non-specific staining, because it does not provide the information such as the molecular weights or sequences of the detected proteins. To verify the specificity for endogenous WT1 in immunohistochemistry, we compared results of WT1-immunohistochemistry with those of western blotting and qRT-PCR in 35 human cell lines. Fig. 4 shows the results of immunohistochemistry, western blotting and qRT-PCR. For immunohistochemistry, immunostaining scores were calculated independently in the entire nucleus, the nuclear bodies and the cytoplasm (Fig. 2). In western blotting, multiple protein bands with different molecular weights were observed; these bands were classified into A-G (Fig. 4B) and their intensities were, respectively, quantified. In qRT-PCR, we used both intercalator-based and fluorescent probe-based methods. Table II shows all quantified results: the immunostaining scores, the intensities of western blotting bands and WT1 mRNA levels.

Table III shows the relationships among quantified results from immunohistochemistry, western blotting and qRT-PCR of the 35 cell lines; positive correlations in significance (both $r>0.4$ and $\mathrm{P}<0.05)$ are indicated in bold. Any relationship among the intensities of western blot bands and WT1 mRNA expression levels yielded strongly positive correlations with statistical significance, with the exception of relationships including western blot band B. The immunostaining scores of the entire nucleus generated by monoclonal $6 \mathrm{~F}-\mathrm{H} 2$ positively correlated with any intensity of western blotting bands other than band B, as well as any WT1 mRNA expression levels. The immunostaining scores of the entire nucleus by monoclonal 


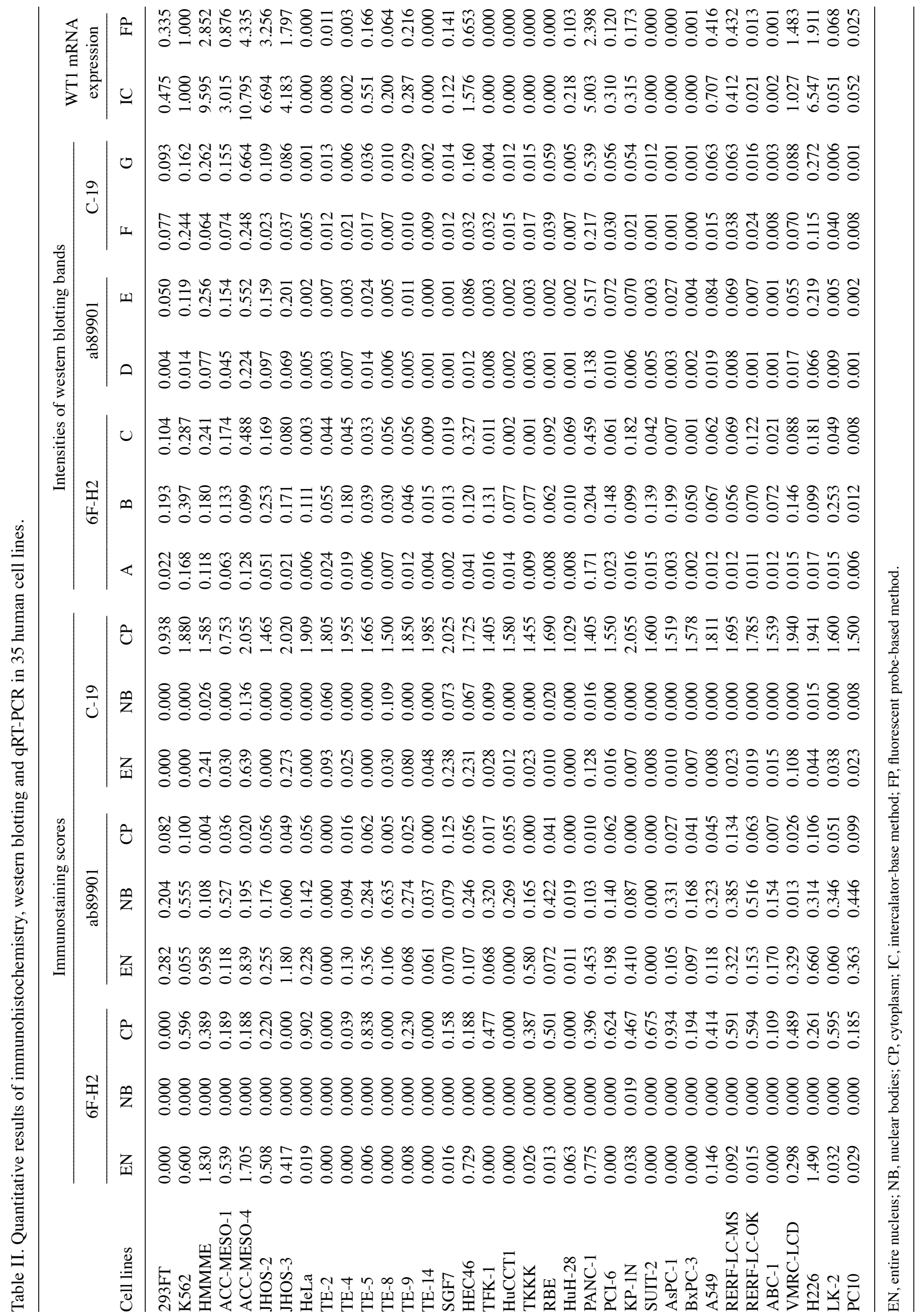




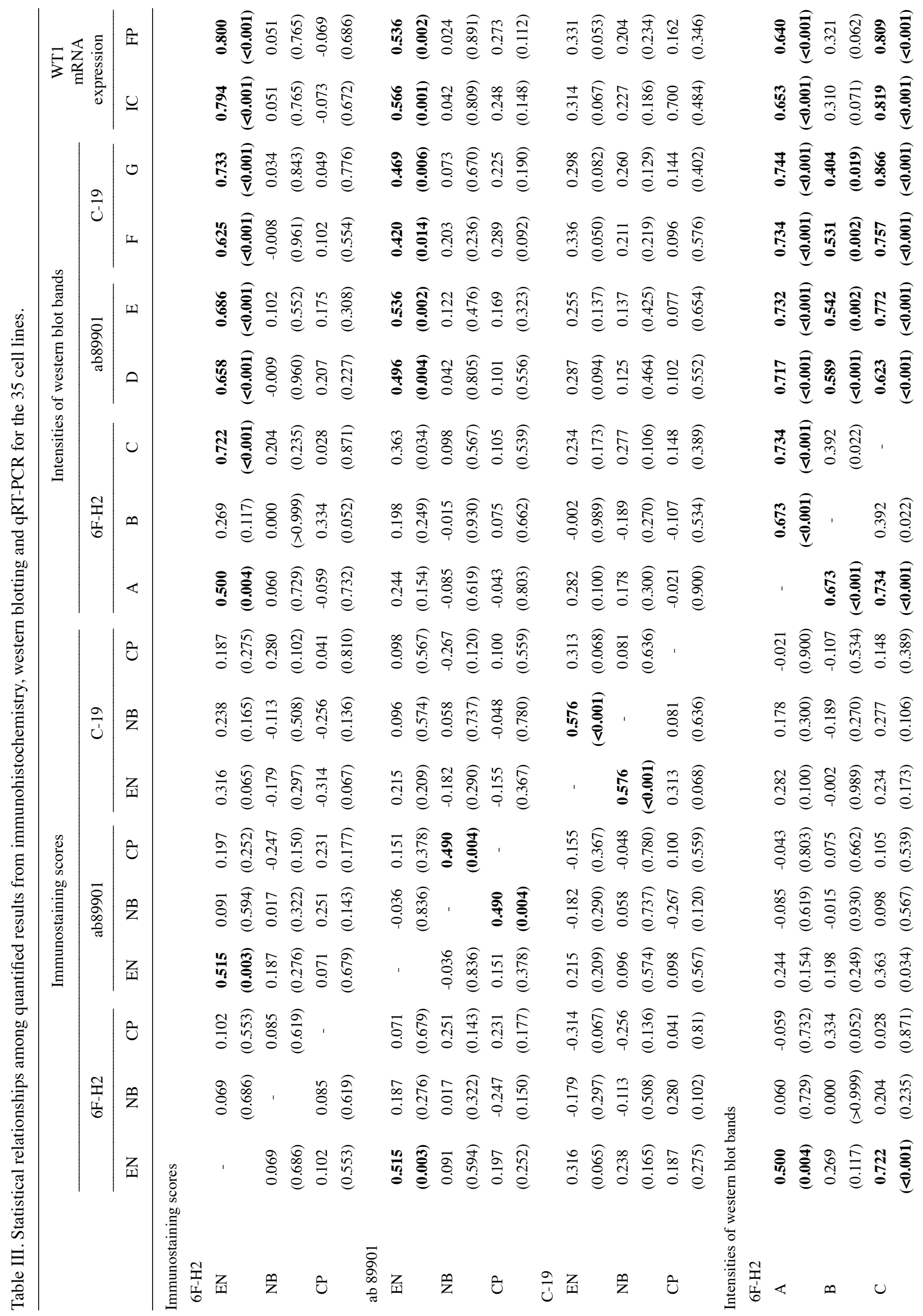




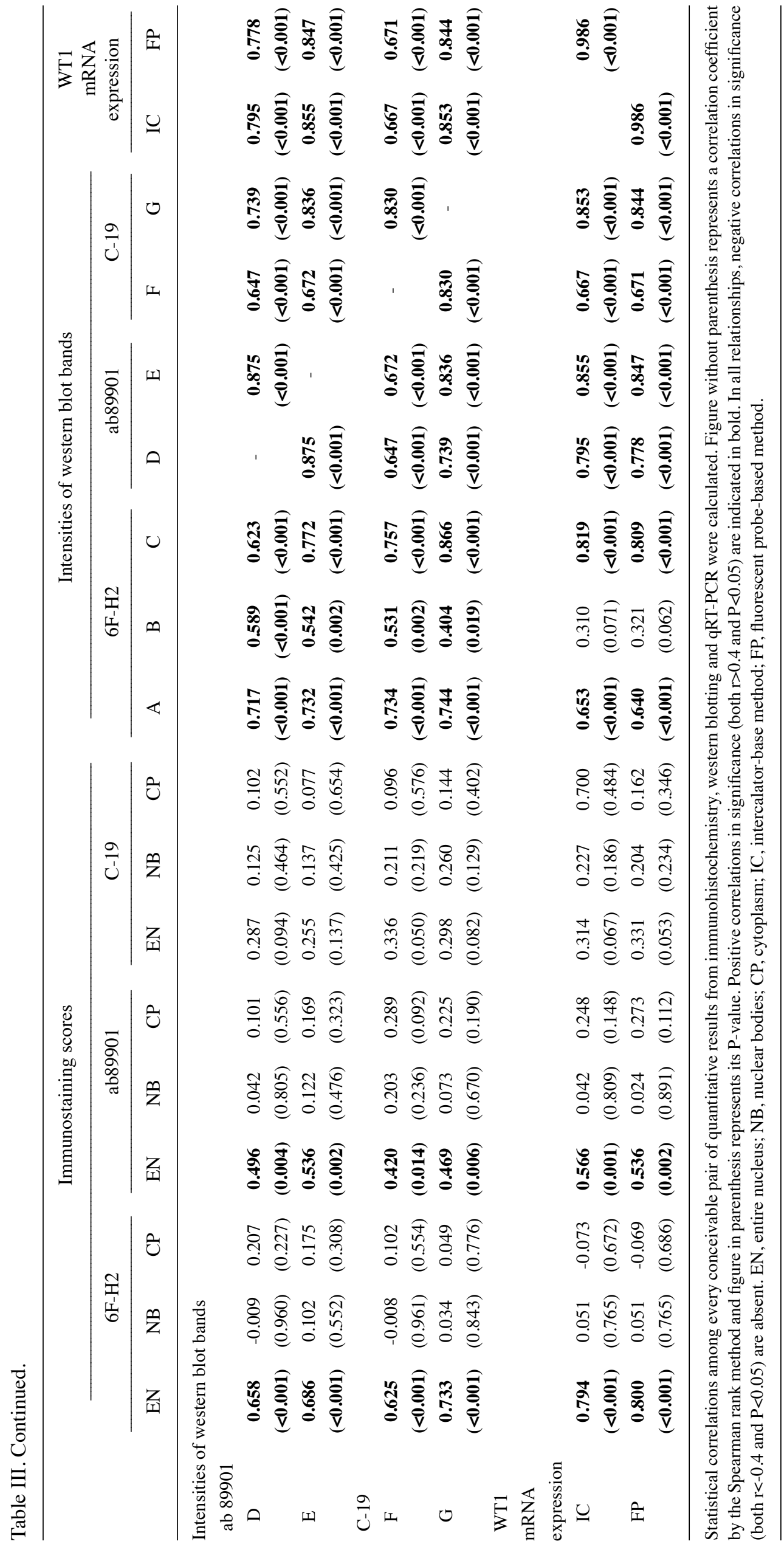




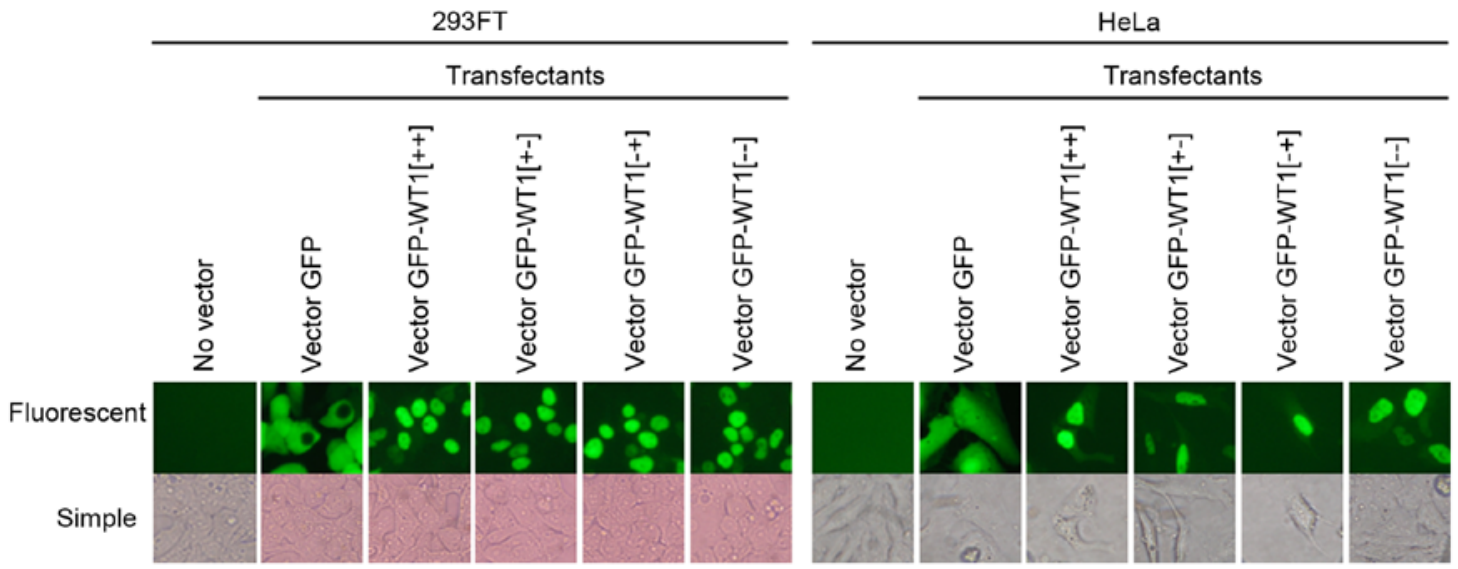

Figure 5. Nuclear localization of GFP-WT1 fusion protein in human cell lines. 293FT and HeLa cells were transfected with vectors encoding GFP, GFP-WT1[++], GFP-WT1[+-], GFP-WT1[-+], or GFP-WT1[--] fusion proteins. The localizations of GFP or GFP-WT1 fusion proteins were visualized by fluorescence microscopy.
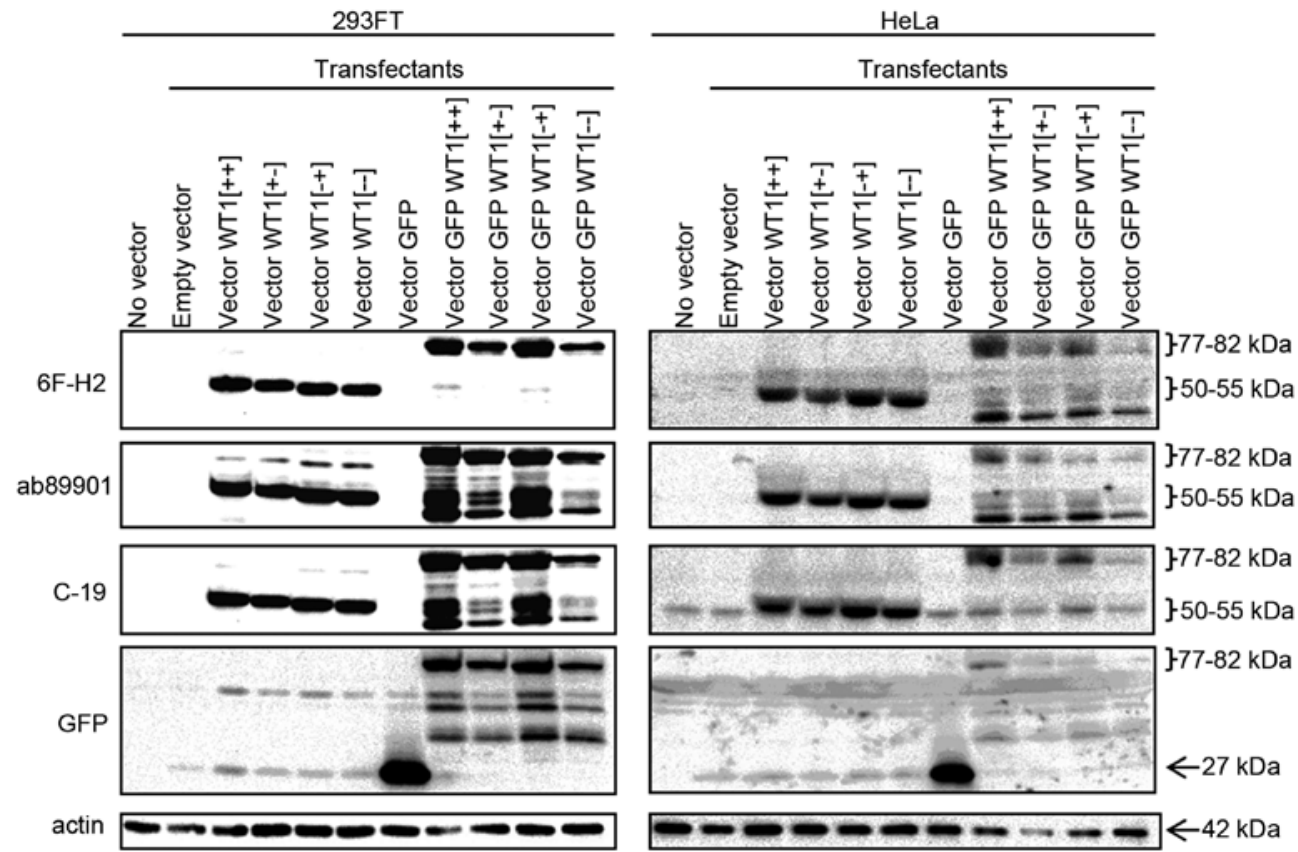

Figure 6. Western blot analysis for GFP-WT1 transfectants. The expected molecular weight of GFP-WT1 fusion protein was 77-82 kDa, because GFP and the four types of WT1 protein are respectively $27 \mathrm{kDa}$ and $50-55 \mathrm{kDa}$ in molecular weight. Western blotting for 293FT and HeLa cells were performed in Condition A and B, respectively.

ab89901 positively correlated with the nuclear immunostaining scores by $6 \mathrm{~F}-\mathrm{H} 2$, intensities of multiple western blot bands, and WT1 mRNA expression levels. By contrast, the immunostaining scores of the entire nucleus by polyclonal C-19 did not correlate with intensities of western blot bands or WT1 mRNA expression levels. No significant correlations were observed between any immunostaining score of the nuclear bodies or cytoplasm generated by three WT1 antibodies and any result of western blotting or qRT-PCR.

These results suggest that western blot bands except for band B and WT1 mRNA expression levels are credible indicators of endogenous WT1 expression. In attunement with them, nuclear immunostaining using 6F-H2 and ab89901 seems to quantitatively reflect endogenous WT1 expression. By contrast, cytoplasmic immunostaining using WT1 antibodies does not specifically reflect WT1 expression because it did not have any significant association with results from other detection methods.

Intracellular localization of GFP-WT1 fusion protein. To verify the intracellular localization of WT1 protein by another method, we examined the localization of GFP-WT1 fusion protein. Fig. 5 shows microscopic images of cells transfected with vector GFP-WT1. While green fluorescence was observed in both the nucleus and the cytoplasm of cells transduced with GFP alone, it was observed only in the nucleus of cells transfected with vector GFP-WT1. Expression of GFP-WT1 fusion protein in the transfectants was confirmed by western blotting (Fig. 6). These results indicate that WT1 protein strongly prefers to be concentrated in the nucleus supporting 


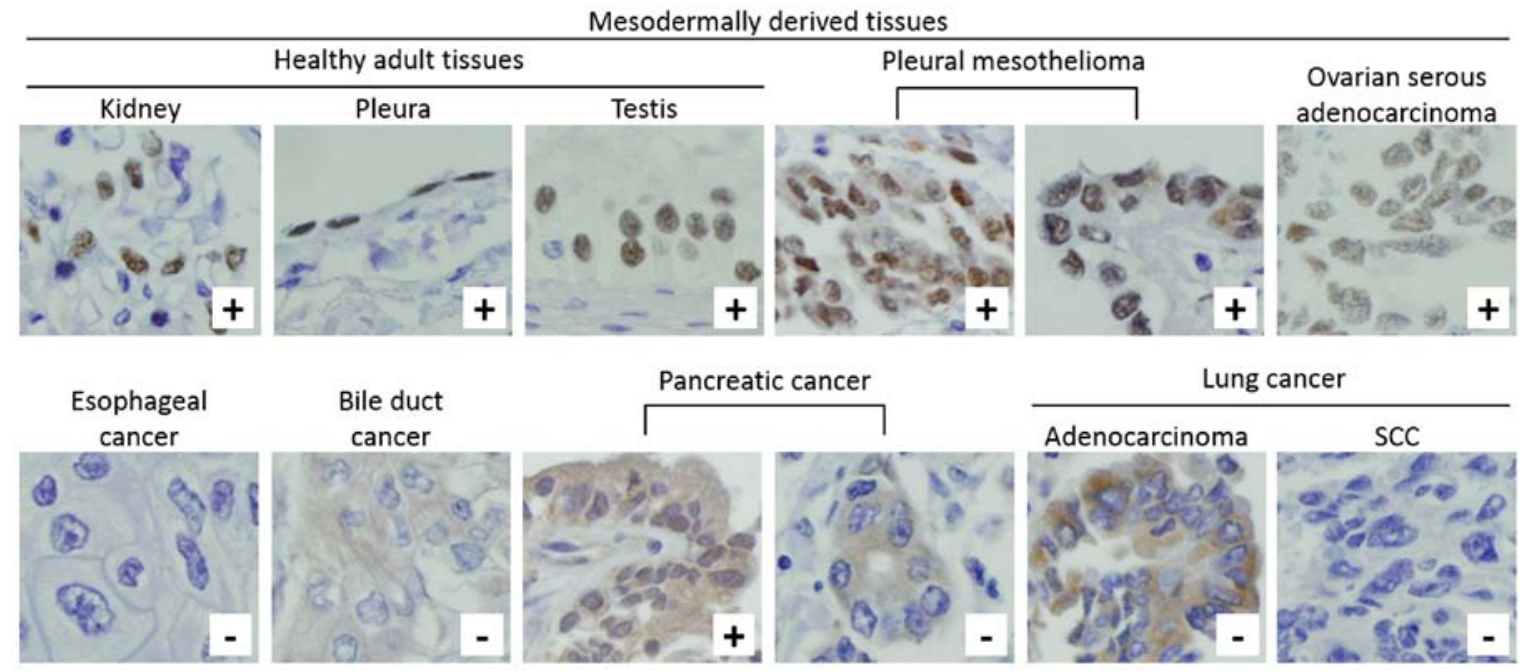

Figure 7. Nuclear immunostaining by $6 \mathrm{~F}-\mathrm{H} 2$ was barely detectable in four types of solid cancers. Representative immunohistochemical staining of healthy and malignant tissue samples using the 6F-H2 monoclonal antibody. '+' represents positive staining of the entire nucleus; '-' represents negative staining of the entire nucleus. SCC, squamous cell carcinoma.

Table IV. Immunohistochemical results from human tissue samples.

Immunostaining $(6 \mathrm{~F}-\mathrm{H} 2)$

\begin{tabular}{lccccc} 
& \multicolumn{2}{c}{ Cancer cells } & & \multicolumn{2}{c}{ Normal epithelium } \\
\cline { 2 - 3 } Human tissue samples & $\begin{array}{c}\text { Entire nucleus } \\
\text { n/total, }(\%)\end{array}$ & $\begin{array}{c}\text { Cytoplasm } \\
\text { n/total, }(\%)\end{array}$ & $\begin{array}{c}\text { Entire nucleus } \\
\text { n/total, }(\%)\end{array}$ & $\begin{array}{c}\text { Cytoplasm } \\
\text { n/total, }(\%)\end{array}$ \\
\hline Esophagus & $0 / 99(0)$ & $79 / 99(80)$ & & $0 / 89(0)$ & $38 / 89(43)$ \\
Bile duct & $0 / 95(0)$ & $59 / 95(62)$ & & $0 / 54(0)$ & $33 / 54(61)$ \\
Pancreas & $3 / 98(3.1)$ & $58 / 98(59)$ & & $1 / 53(1.9)$ & $5 / 53(9.4)$ \\
Lung & & & & & \\
(Adenocarcinoma) & $0 / 164(0)$ & $83 / 164(51)$ & & $0 / 158(0)$ & $0 / 158(0)$ \\
(Squamous cell carcinoma) & $0 / 71(0)$ & $33 / 71(46)$ & & $0 / 61(0)$ & $0 / 61(0)$ \\
(Cancers of other histological types) & $0 / 14(0)$ & $5 / 14(36)$ & & $0 / 11(0)$ & $0 / 11(0)$ \\
\hline
\end{tabular}

the idea that nuclear immunostaining quantitatively reflects WT1 expression.

WT1 expression in human tissue samples. We examined WT1 expression in primary cancer tissue samples of esophagus, bile duct, pancreas and lung with the corresponding normal epithelial tissues by immunohistochemistry using $6 \mathrm{~F}-\mathrm{H} 2$, an antibody shown above to be appropriate for WT1-immunohistochemistry. As shown in Fig. 7 and Table IV, nuclear immunostaining was almost never detected in cancer cells or normal epithelium of esophagus, bile duct, pancreas, and lung, whereas it was firmly observed in mesothelioma, ovarian cancer and healthy tissues such as kidney and pleura.

\section{Discussion}

In the present study, we attempted to validate WT1-immunohistochemistry for solid tumors quantifying WT1 gene products. Utilizing multiple different detection methods and WT1 antibodies, along with defined positive and negative controls, we revealed that only nuclear staining by immunohistochemistry using appropriate WT1-specific antibodies positively correlates, in a statistically significant manner, with the intensities of western blot bands of defined molecular weights and with mRNA levels determined by qRT-PCR. In contrast, the cytoplasmic immunostaining is considered to be non-specific because it did not correlate with any results from other specific examinations. The nuclear immunostaining was hardly observed in primary esophageal, bile duct, pancreatic, or lung cancer although it was firmly observed in mesodermally derived tissues. These results indicate that WT1 expression in those non-mesodermal solid cancers is imperceptible compared to mesodermal tissues. On the other hand, cytoplasmic immunostaining was frequently observed in those solid cancers and WT1 would be estimated to be overexpressed in those solid cancers if researchers consider the cytoplasmic staining as WT1 expression.

Researchers involved in WT1-targeted treatment of cancer presume that WT1 is overexpressed in various malignancies including non-mesodermal solid cancers (17-28). There is 
little controversy regarding the observation that solid cancers of non-mesodermal origin rarely show nuclear immunostaining but frequently exhibit cytoplasmic immunostaining, while mesodermal tissues provide the nuclear immunostaining $(8-16,18-24,28)$. Differences of the conclusions about WT1 expression in non-mesodermal solid cancers stem largely from a discrepancy in interpretations of the cytoplasmic immunostaining. Our conclusion is supported by many pathological studies. Pathologists generally consider the cytoplasmic immunostaining for WT1 as non-specific reaction; they have described that WT1 is hardly expressed in nonmesodermal solid cancers such as colon cancer $(0 \%)(11,15)$, pancreatic cancer $(0 \%)(16)$, thyroid cancer $(0 \%)(11)$, prostate cancer $(0 \%)(11)$, lung cancer $(0-20 \%)(8-15)$ and breast cancer $(0-7 \%)(11,13,15)$. The pathologists thus use WT1 to distinguish mesothelioma from lung cancers $(8,10-13)$, or ovarian cancer cells from pancreatic cancer cells in malignant ascites (16). The present study scientifically supports the pathologists to judge the cytoplasmic immunostaining for WT1 as non-specific.

We validated WT1-immunohistochemistry in human cell lines using multiple detection methods but examined WT1 expression in human primary tissues only by immunohistochemistry. It is desirable to present results of western blotting and qRT-PCR in tissue samples because subcellular localization of WT1 protein can differ between cell lines and tissues. However, it should be noted that tissue samples inevitably contain some fraction of stroma and blood; these tissues should not be ignored because they can include cell types that express WT1. Contamination of tumor samples by non-tumor tissues is particularly important in qRT-PCR. Furthermore, because of intra-tumor heterogeneity, samples from different sites of the same cancer do not always exhibit consistent protein expression (42). On another front, the evaluation of WT1 in the fractionated organelle may be performed when one intends to approach the question regarding to the cytoplasmic WT1. However, the quantitative evaluation of WT1 protein in the cytoplasmic fraction seems to be hard because of the technical difficulty of avoiding the undesired contamination from other fractions. WT1 has multiple isoforms other than the four types of isoforms focused on in the present study; the present study cannot deny the possibility that alternative WT1 transcripts mainly localize in the cytoplasm.

Although the results were obtained from the limited study as described above, they suggest that cytoplasmic staining in immunohistochemistry for WT1 does not reflect actual WT1 expression, WT1 is not overexpressed in non-mesodermal solid cancers, and researchers need to reconsider whether WT1 is an appropriate target of treatment for patients with nonmesodermal solid cancers or not. It is definite that qRT-PCR cannot detect several kinds of WT1 splice variants due to restrictions by the primers. However, western blotting using multiple WT1-specific antibodies is expected to detect more kinds of WT1 splice variants than qRT-PCR and it is not negligible that the cytoplasmic immunostaining did not correlate with western blotting although the nuclear immunostaining did. Because only nuclear immunostaining by WT1-specific monoclonal antibodies correlated with western blotting using the same antibodies, non-coordinate cytoplasmic immunostaining cannot be a reliable indicator of WT1 expression regardless of other putative WT1 splice variants. We do not exclude the possibility that a small amount of WT1 protein may exist in the cytoplasm and the small quantity of WT1 protein can be enough for WT1-targeted immunotherapy to work. Investigating whether WT1-targeted treatment of cancer specifically works for human cells with no nuclear immunostaining but considerable cytoplasmic immunostaining would provide critical information to the problem.

\section{References}

1. Call KM, Glaser T, Ito CY, Buckler AJ, Pelletier J, Haber DA, Rose EA, Kral A, Yeger H, Lewis WH, et al: Isolation and characterization of a zinc finger polypeptide gene at the human chromosome 11 Wilms' tumor locus. Cell 60: 509-520, 1990.

2. Gessler M, Poustka A, Cavenee W, Neve RL, Orkin SH and Bruns GA: Homozygous deletion in Wilms tumours of a zincfinger gene identified by chromosome jumping. Nature 343: 774-778, 1990.

3. Pritchard-Jones K, Fleming S, Davidson D, Bickmore W, Porteous D, Gosden C, Bard J, Buckler A, Pelletier J, Housman D, et al: The candidate Wilms' tumour gene is involved in genitourinary development. Nature 346: 194-197, 1990.

4. Mundlos S, Pelletier J, Darveau A, Bachmann M, Winterpacht A and Zabel B: Nuclear localization of the protein encoded by the Wilms' tumor gene WT1 in embryonic and adult tissues. Development 119: 1329-1341, 1993.

5. Larsson SH, Charlieu JP, Miyagawa K, Engelkamp D, Rassoulzadegan M, Ross A, Cuzin F, van Heyningen V and Hastie ND: Subnuclear localization of WT1 in splicing or transcription factor domains is regulated by alternative splicing. Cell 81: 391-401, 1995.

6. Menssen HD, Renkl HJ, Rodeck U, Maurer J, Notter M, Schwartz S, Reinhardt R and Thiel E: Presence of Wilms' tumor gene (wt1) transcripts and the WT1 nuclear protein in the majority of human acute leukemias. Leukemia 9: 1060-1067, 1995.

7. Miwa H, Beran M and Saunders GF: Expression of the Wilms' tumor gene (WT1) in human leukemias. Leukemia 6: 405-409, 1992.

8. Amin KM, Litzky LA, Smythe WR, Mooney AM, Morris JM, Mews DJ, Pass HI, Kari C, Rodeck U, Rauscher FJ III, et al: Wilms' tumor 1 susceptibility (WT1) gene products are selectively expressed in malignant mesothelioma. Am J Pathol 146: 344-356, 1995.

9. Kumar-Singh S, Segers K, Rodeck U, Backhovens H, Bogers J, Weyler J, Van Broeckhoven C and Van Marck E: WT1 mutation in malignant mesothelioma and WT1 immunoreactivity in relation to p53 and growth factor receptor expression, cell-type transition, and prognosis. J Pathol 181: 67-74, 1997.

10. Oates J and Edwards C: HBME-1, MOC-31, WT1 and calretinin: An assessment of recently described markers for mesothelioma and adenocarcinoma. Histopathology 36: 341-347, 2000.

11. Ordóñez NG: Value of thyroid transcription factor-1, E-cadherin, BG8, WT1, and CD44S immunostaining in distinguishing epithelial pleural mesothelioma from pulmonary and nonpulmonary adenocarcinoma. Am J Surg Pathol 24: 598-606, 2000.

12. Foster MR, Johnson JE, Olson SJ and Allred DC: Immunohistochemical analysis of nuclear versus cytoplasmic staining of WT1 in malignant mesotheliomas and primary pulmonary adenocarcinomas. Arch Pathol Lab Med 125: 1316-1320, 2001.

13. Hecht JL, Lee BH, Pinkus JL and Pinkus GS: The value of Wilms tumor susceptibility gene 1 in cytologic preparations as a marker for malignant mesothelioma. Cancer 96: 105-109, 2002.

14. Tsuta K, Kato Y, Tochigi N, Hoshino T, Takeda Y, Hosako M, Maeshima AM, Asamura H, Kondo T and Matsuno Y: Comparison of different clones (WT49 versus 6F-H2) of WT-1 antibodies for immunohistochemical diagnosis of malignant pleural mesothelioma. Appl Immunohistochem Mol Morphol 17: 126-130, 2009.

15. Hwang H, Quenneville L, Yaziji $\mathrm{H}$ and Gown AM: Wilms tumor gene product: Sensitive and contextually specific marker of serous carcinomas of ovarian surface epithelial origin. Appl Immunohistochem Mol Morphol 12: 122-126, 2004.

16. Han L, Pansare V, Al-Abbadi M, Husain $M$ and Feng J: Combination of MUC5ac and WT-1 immunohistochemistry is useful in distinguishing pancreatic ductal carcinoma from ovarian serous carcinoma in effusion cytology. Diagn Cytopathol 38: 333-336, 2010. 
17. Cheever MA, Allison JP, Ferris AS, Finn OJ, Hastings BM, Hecht TT, Mellman I, Prindiville SA, Viner JL, Weiner LM, et al: The prioritization of cancer antigens: A National Cancer Institute pilot project for the acceleration of translational research. Clin Cancer Res 15: 5323-5337, 2009.

18. Oji Y, Yano M, Nakano Y, Abeno S, Nakatsuka S, Ikeba A, Yasuda T, Fujiwara Y, Takiguchi S, Yamamoto $\mathrm{H}$, et al: Overexpression of the Wilms' tumor gene WT1 in esophageal cancer. Anticancer Res 24 (5B): 3103-3108, 2004.

19. Nakatsuka S, Oji Y, Horiuchi T, Kanda T, Kitagawa M, Takeuchi T, Kawano K, Kuwae Y, Yamauchi A, Okumura M, et al: Immunohistochemical detection of WT1 protein in a variety of cancer cells. Mod Pathol 19: 804-814, 2006.

20. Oji Y, Yamamoto H, Nomura M, Nakano Y, Ikeba A, Nakatsuka S, Abeno S, Kiyotoh E, Jomgeow T, Sekimoto M et al: Overexpression of the Wilms' tumor gene WT1 in colorectal adenocarcinoma. Cancer Sci 94: 712-717, 2003.

21. Sera T, Hiasa Y, Mashiba T, Tokumoto Y, Hirooka M, Konishi I, Matsuura B, Michitaka K, Udaka K and Onji M: Wilms' tumour 1 gene expression is increased in hepatocellular carcinoma and associated with poor prognosis. Eur J Cancer 44: 600-608, 2008

22. Oji Y, Nakamori S, Fujikawa M, Nakatsuka S, Yokota A, Tatsumi N, Abeno S, Ikeba A, Takashima S, Tsujie M, et al: Overexpression of the Wilms' tumor gene WT1 in pancreatic ductal adenocarcinoma. Cancer Sci 95: 583-587, 2004.

23. Oji Y, Miyoshi Y, Koga S, Nakano Y, Ando A, Nakatsuka S, Ikeba A, Takahashi E, Sakaguchi N, Yokota A, et al: Overexpression of the Wilms' tumor gene WT1 in primary thyroid cancer. Cancer Sci 94: 606-611, 2003.

24. Oji Y, Miyoshi S, Maeda H, Hayashi S, Tamaki H, Nakatsuka S, Yao M, Takahashi E, Nakano Y, Hirabayashi $\mathrm{H}$, et al: Overexpression of the Wilms' tumor gene WT1 in de novo lung cancers. Int J Cancer 100: 297-303, 2002.

25. Loeb DM, Evron E, Patel CB, Sharma PM, Niranjan B, Buluwela L, Weitzman SA, Korz D and Sukumar S: Wilms tumor suppressor gene (WT1) is expressed in primary breast tumors despite tumor-specific promoter methylation. Cancer Res 61: 921-925, 2001

26. Miyoshi Y, Ando A, Egawa C, Taguchi T, Tamaki Y, Tamaki H, Sugiyama H and Noguchi S: High expression of Wilms' tumor suppressor gene predicts poor prognosis in breast cancer patients. Clin Cancer Res 8: 1167-1171, 2002.

27. Lee AH, Paish EC, Marchio C, Sapino A, Schmitt FC, Ellis IO and Reis-Filho JS: The expression of Wilms' tumour-1 and Ca125 in invasive micropapillary carcinoma of the breast Histopathology 51: 824-828, 2007.

28. Oji Y, Suzuki T, Nakano Y, Maruno M, Nakatsuka S, Jomgeow T, Abeno S, Tatsumi N, Yokota A, Aoyagi S, et al: Overexpression of the Wilms' tumor gene W T1 in primary astrocytic tumors. Cancer Sci 95: 822-827, 2004.

29. Oka Y, Tsuboi A, Taguchi T, Osaki T, Kyo T, Nakajima H, Elisseeva OA, Oji Y, Kawakami M, Ikegame K, et al: Induction of WT1 (Wilms' tumor gene)-specific cytotoxic T lymphocytes by WT1 peptide vaccine and the resultant cancer regression. Proc Natl Acad Sci USA 101: 13885-13890, 2004

30. Morita S, Oka Y, Tsuboi A, Kawakami M, Maruno M, Izumoto S, Osaki T, Taguchi T, Ueda T, Myoui A, et al: A phase I/II trial of a WT1 (Wilms' tumor gene) peptide vaccine in patients with solid malignancy: Safety assessment based on the phase I data. Jpn J Clin Oncol 36: 231-236, 2006
31. Izumoto S, Tsuboi A, Oka Y, Suzuki T, Hashiba T, Kagawa N, Hashimoto N, Maruno M, Elisseeva OA, Shirakata T, et al: Phase II clinical trial of Wilms tumor 1 peptide vaccination for patients with recurrent glioblastoma multiforme. J Neurosurg 108: 963-971, 2008.

32. Keilholz U, Letsch A, Busse A, Asemissen AM, Bauer S, Blau IW, Hofmann WK, Uharek L, Thiel E and Scheibenbogen C: A clinical and immunologic phase 2 trial of Wilms tumor gene product 1 (WT1) peptide vaccination in patients with AML and MDS. Blood 113: 6541-6548, 2009.

33. Ohno S, Kyo S, Myojo S, Dohi S, Ishizaki J, Miyamoto K, Morita S, Sakamoto J, Enomoto T, Kimura T, et al: Wilms' tumor 1 (WT1) peptide immunotherapy for gynecological malignancy. Anticancer Res 29: 4779-4784, 2009.

34. Van Tendeloo VF, Van de Velde A, Van Driessche A, Cools N, Anguille S, Ladell K, Gostick E, Vermeulen K, Pieters K, Nijs G, et al: Induction of complete and molecular remissions in acute myeloid leukemia by Wilms' tumor 1 antigen-targeted dendritic cell vaccination. Proc Natl Acad Sci USA 107: 13824-13829, 2010.

35. Krug LM, Dao T, Brown AB, Maslak P, Travis W, Bekele S, Korontsvit T, Zakhaleva V, Wolchok J, Yuan J, et al: WT1 peptide vaccinations induce CD4 and CD8 T cell immune responses in patients with mesothelioma and non-small cell lung cancer. Cancer Immunol Immunother 59: 1467-1479, 2010.

36. Kaida M, Morita-Hoshi Y, Soeda A, Wakeda T, Yamaki Y, Kojima Y, Ueno H, Kondo S, Morizane C, Ikeda M, et al: Phase 1 trial of Wilms tumor 1 (WT1) peptide vaccine and gemcitabine combination therapy in patients with advanced pancreatic or biliary tract cancer. J Immunother 34: 92-99, 2011.

37. Usami N, Fukui T, Kondo M, Taniguchi T, Yokoyama T, Mori S, Yokoi K, Horio Y, Shimokata K, Sekido Y, et al: Establishment and characterization of four malignant pleural mesothelioma cell lines from Japanese patients. Cancer Sci 97: 387-394, 2006.

38. Nishihira T, Hashimoto Y, Katayama M, Mori S and Kuroki T: Molecular and cellular features of esophageal cancer cells. J Cancer Res Clin Oncol 119: 441-449, 1993.

39. Saito T, Kato H, Saito M, Karaki Y, Tazawa K and Fujimaki M: TNF receptor number-dependent cytotoxicity to TNF-resistant human esophageal cancer cell lines by combination with recombinant human necrosis factor and hyperthermia. Hum Cell 7: 55-61, 1994.

40. Yanagihara K, Ito A, Toge T and Numoto M: Antiproliferative effects of isoflavones on human cancer cell lines established from the gastrointestinal tract. Cancer Res 53: 5815-5821, 1993.

41. Sugiura H, Ishikura H, Omi M, Kaji M, Iwai K, Kishimoto T, Takahashi T, Kimura C, Kato H and Yoshiki T: Lymphokineactivated killer cytotoxicity against pancreas adenocarcinoma cell lines and vascular endothelial cells. Pathol Int 44: 688-696, 1994.

42. Gerlinger M, Rowan AJ, Horswell S, Larkin J, Endesfelder D, Gronroos E, Martinez P, Matthews N, Stewart A, Tarpey P, et al: Intratumor heterogeneity and branched evolution revealed by multiregion sequencing. N Engl J Med 366: 883-892, 2012. 